\title{
Diacronie
}

Studi di Storia Contemporanea

$\mathrm{N}^{\circ} 15,3 \mid 2013$

Spazi, percorsi e memorie

\section{Il cammino delle infermiere verso la professionalizzazione}

María Ángeles Hijano Pérez

\section{(2) OpenEdition}

Journals

\section{Edizione digitale}

URL: http://journals.openedition.org/diacronie/600

DOI: 10.4000/diacronie.600

ISSN: 2038-0925

\section{Editore}

Association culturelle Diacronie

\section{Notizia bibliografica digitale}

María Ángeles Hijano Pérez, « Il cammino delle infermiere verso la professionalizzazione », Diacronie [Online], N 15, 3 | 2013, documento 14, Messo online il 01 octobre 2013, consultato il 02 mai 2019. URL : http://journals.openedition.org/diacronie/600 ; DOI : 10.4000/diacronie.600 


\section{Diacronie}

N. 15 | 3|2013 Spazi, percorsi e memorie

\section{4/}

\section{Il cammino delle infermiere verso la professionalizzazione}

María Ángeles HIJANO PÉREZ*

In questo articolo vogliamo esaminare le strategie messe in atto dalle infermiere spagnole affinché la loro attività non fosse vista unicamente come un'opera filantropica, ma venisse invece considerata un'importante pratica professionale durante i conflitti bellici che colpivano l'Europa. Per tale ragione, in primo luogo esamineremo la situazione delle infermiere spagnole verso la fine del secolo XIX, per poi analizzare i fattori che contribuirono alla creazione delle scuole di infermieristica in Spagna, passo imprescindibile verso la professionalizzazione. La monarchia e la Croce Rossa [Cruz Roja] furono le due istituzioni che, strettamente legate tra loro, contribuirono al processo di conversione dell'occupazione delle infermiere in una professione. 
Il ministro inglese ha attraversato campi e città, aziende agricole e fabbriche di munizioni, campi di prigionia ed ospedali per i feriti, uffici postali e sale adibite alla realizzazione di uniformi militari. In tutti questi luoghi ha verificato l'eroica serenità femminile. Ha visto la donna sostituire l'uomo senza alcuno sforzo, né lamentela. Allevatrici, veterinarie, infermiere, postine, granatiere, tranviere, controllori di treni, sentinelle delle prigioni.

Cristóbal Castro, 14 gennaio $1916^{1}$.

\section{Introduzione}

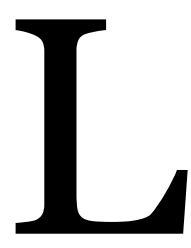

a citazione a cui si fa riferimento, pubblicata nel 1916 nella revista «Nuevo Mundo» di Madrid, riprende un discorso del ministro britannico Lloyd George, nel quale si elogiava l'importanza delle donne lavoratrici per far progredire gran parte dei paesi europei, piegati allora dalla guerra. In Spagna, neutrale durante la Grande Guerra, una volta terminato il conflitto non si produsse la stessa spinta verso la professionalizzazione delle donne; ciononostante, in qualche modo, coloro che si erano dedicate alla cura degli ammalati si allontanarono progressivamente dalle pratiche filantropiche per incamminarsi, seppure in modo più graduale e tardivo rispetto alla maggior parte d'Europa, verso la professionalizzazione. In questo articolo vogliamo esaminare le strategie messe in atto dalle infermiere spagnole, affinchè la loro attività non fosse vista unicamente come un'opera filantropica, ma venisse invece considerata un'importante pratica professionale durante i conflitti bellici che colpivano l'Europa. Per tale ragione, in primo luogo esamineremo la situazione delle infermiere spagnole sul finire del secolo XIX, per poi analizzare i fattori che contribuirono alla creazione delle scuole di infermieristica in Spagna, passo imprescindibile verso la professionalizzazione. La monarchia e la Cruz Roja furono le due istituzioni che, strettamente collegate tra loro, contribuirono al processo di conversione dell'occupazione delle infermiere, in professione ${ }^{2}$.

${ }^{1}$ CASTRO, Cristobal, «Las mujeres. Fortaleza del sexo débil», in Nuevo Mundo, 14 gennaio 1916, p. 24.

2 Estatutos de la Cruz Roja Española (aprobados por R.O. circular del Ministerio de la Guerra a 16 de junio de 1917), Madrid, ed. oficial de la Asamblea Suprema, 1922. Nel testo si può leggere: «Tale Istituzione, dichiarata di utilità e beneficio per tutto il territorio della monarchia, e unica autorizzata ufficialmente all'assistenza ai feriti di guerra, ha capacità giuridica in ambito civile $[\ldots]$ » [le traduzioni dallo spagnolo sono a cura dell'autrice]. 


\section{Un argomento sconosciuto}

Fra le prime opere che menzionano la professione infermieristica in Spagna, realizzate da autrici come Concepción Arenal, una delle maggiori attiviste per i diritti femminili, si misero in evidenza le difficoltà che attraversava il settore, specialmente per quanto riguardasse il ruolo delle donne. La grande preparazione intellettuale di cui godeva Arenal, le permise di sottolineare le tremende ingiustizie che la donna spagnola era costretta ad affrontare per ottenere una remunerazione pari a quella dell'uomo3.

L'autrice fu tra coloro che maggiormente si batterono per ottenere l'integrazione della donna nel mondo infermieristico. Partendo da una prospettiva romantica, sosteneva che le donne con un'adeguata preparazione avrebbero potuto imparare ad esercitare un lavoro strettamente connesso alla serietà e all'affetto, qualità queste, secondo la Arenal, per natura prettamente femminili. Per tale ragione, ancor prima che venissero fondate le prime scuole di infermeristica, Concepción Arenal già si faceva portavoce di una serie di principi che dovevano essere considerati da tutte coloro che avessero avuto intenzione di dedicarsi a questo settore: serietà nell'assistenza, conoscenze in materia di alimentazione, rettitudine, pulizia ed ordine. Principi altruistici che ogni infermiera doveva tenere presenti, per trasformare il lavoro in professione4.

Arenal era convinta che permanessero disuguaglianze tra generi, favorite dalle leggi stesse, arrivando ad affermare che «La legge proibisce alla donna l'esercizio di ogni professione: solo recentemente si è giunti a ritenerla idonea per l'insegnamento di base per le bambine»5. Agli inizi degli anni Settanta dell'Ottocento, grazie a questo interesse dimostrato nei confronti della condizione delle donne, la scrittrice e filantropa spagnola fu nominata Segretaria Generale della Croce Rossa di Madrid, dandole la possibilità di

\footnotetext{
3 ARENAL, Concepción, La emancipación de la mujer en España, Madrid, Ed. Jucar, 1974, con edizione e prologo di Mauro Armiño. In questo libro viene recuperata una selezione di testi dell'autrice: qui tuttavia ancora non appare la difesa dell'infermieristica che affronteremo più avanti.

4 «Si crea un Corpo di Infermiere Professioniste della Croce Rossa, con lo scopo che tale Istituzione possa disporre di personale avente un'istruzione sufficiente per il servizio interno dei suoi Ospedali, e che allo stesso tempo permetta a tali infermiere, grazie alle conoscenze acquisite, di esercitare la professione sia nella cura diretta degli ammalati tramite visite a domicilio, sia nell'assistere il personale medico che richiedesse i loro servizi». "Creación del Cuerpo de Enfermeras Profesionales de la Cruz Roja Española. Real Orden de 26 de octubre de 1922", in Diario Oficial del Ministerio de la Guerra, 242, 28 ottobre 1922, p. 403.

5 ARENAL, Concepción, La emancipación de la mujer en España, cit., p. 128.
} 
creare, nel 1874, un gruppo sanitario di infermiere e medici per l'assistenza ai feriti causati dalla Terza Guerra Carlista (1872- 1876) ${ }^{6}$.

In questo panorama, Concepción Arenal fu evidentemente un'antesignana di quell'opera altruista che invitava le donne ad offrire aiuto ed assistenza alle associazioni per i feriti dei conflitti bellici. In Spagna, per esempio, collaborò organizzando «La Croce Rossa del Soccorso», rivolta ai feriti delle Guerre Carliste7. Shirley Manzini, nel suo libro in cui presenta alcune delle grandi donne spagnole, delinea una rapida biografia di Concepción Arenal e ricorda che durante la Terza Guerra Carlista accompagnò le truppe al campo di battaglia di Miranda del Ebro a cavallo di un asino per poter vedere uno dei suoi figli, ufficiale in servizio nel corso di quell'evento bellico ${ }^{8}$. La sua opera come avvocato penalista, sociologa e scrittrice, la rende una figura esemplare nella lotta contro le disuguaglianze, nonostante la conducesse da un punto di vista prettamente cattolico e difficilmente definibile come rivoluzionario ${ }^{\text {. }}$

Il giurista spagnolo Adolfo Posada, autore del libro Feminismo ${ }^{10}$, fu una delle personalità più rilevanti che si dedicarono allo studio dei diritti delle donne; nonostante nella sua opera fosse lecito aspettarsi una valutazione sul ruolo delle infermiere, queste non furono nemmeno ricordate nel capitolo dedicato all'ambito sanitario. Riteneva che le donne avessero svolto ogni tipo di lavoro sociale, e per tale ragione dedicò una sezione ad ognuna delle professioni che considerava rivestissero maggiore importanza. Per descrivere il ruolo occupato dalle donne in tali settori, si limitò a quelle regolamentate dallo Stato, dimenticandone altre, inerenti per esempio alla letteratura, le Belle Arti, il teatro ed il giornalismo. Assegnò un capitolo alla Condizione sociale

${ }^{6}$ Si veda: CLEMENTE, Josep Carles, Historia de una iniciativa humanitaria de la Cruz Roja Española (1918-1997). La Escuela Universitaria de Enfermeras de Madrid, Madrid, Fundamentos-Cruz Roja Española, 1999, p. 55. Una minuziosa analisi dell'infermieristica in Spagna, dove Concepción Arenal viene considerata come precorritrice nell'opera di difesa delle classi sofferenti ed emarginate: il ferito di guerra, il povero o il prigioniero.

7 ARENAL, Concepción, La beneficencia, la filantropía y la caridad, Madrid, Victoriano Suárez, 1927. In questa pubblicazione espone i suoi progetti per estendere alla Spagna le Leggi di beneficenza e di riconoscimento dei diritti della donna. Lo sforzo dimostrato nella creazione di una rete associativa ci presenta un'immagine della sua originalità e creatività, finalizzata a ottenere risultati a beneficio delle classi maggiormente escluse, tra le quali, appunto, le donne. Un ottimo studio biografico sull'autrice è quello di CRUZ ROMEO MATEO, María, Concepción Arenal: reformar la sociedad desde los márgenes, in LEDESMA PÉREZ, Manuel, BURDIEL, Isabel (eds.), Liberales eminentes, Madrid, Marcial Pons, 2008, pp. 213-243.

8 MANGINI, Shirley, Las modernas de Madrid. Las grandes intelectuales españolas de la vanguardia, Barcelona, Península, 2001, p. 40. L'autrice espone le molteplici innovazioni introdotte nella società spagnola da Concepción Arenal, la quale, oltre a una strenue difesa della professionalità della donna, si fa portavoce di comportamenti profondamente moderni per l'epoca.

9 CLEMENTE, Josep Carles, La Escuela Universitaria de Enfermeras de Madrid. Historia de una iniciativa humanitaria de la Cruz Roja Española (1918-1997), Madrid, Fundamentos-Cruz Roja Española, 1999, pp. 55-57.

10 POSADA, Adolfo, Feminismo, Madrid, Cátedra, 1994. 
della donna. Le professioni, con sezioni dedicate a La donna e linsegnamento, La donna e la Medicina e La donna e le professioni di Diritto ${ }^{11}$. Sosteneva che non fosse corretto precludere a priori la strada alle donne, ma quando pubblicò il suo scritto le infermiere non avevano ancora il riconoscimento che puntavano ad ottenere e continuavano ad occupare un posto molto più basso della scala sociale rispetto a quello che poteva spettar loro in considerazione dell'attività che svolgevano.

Nonostante in Spagna non si mostrasse interesse per il lavoro delle infermiere, né si pubblicasse alcunché su di loro, negli Stati Uniti accadeva qualcosa di molto diverso; ne abbiamo testimonianza da alcune pubblicazioni dell'epoca, realizzate da autrici che molto si spesero per questo tema e per il riconoscimento degli altri diritti della donna.

Siamo a conoscenza dell'esistenza di un'opera di Mary Putnam Jacobi (1842-1906), intitolata Woman in medicine, compresa nel libro Woman's work in America ${ }^{12}$, di cui fu editrice Annie Nathan Meyer (1867-1951) e la cui introduzione venne curata da Julia Ward Howe (1819-1910), presidentessa di una delle associazioni che, dal 1869, lottava per ottenere il voto a favore delle donne negli Stati Uniti ${ }^{13}$. Questo libro ospitò scritti di almeno dieci donne, che trattarono diversi temi inerenti il rapporto tra la donna e l'educazione, la letteratura, il giornalismo, la Chiesa, il sistema giuridico, lo Stato, l'industria, la medicina e la filantropia. Nell'ultima sezione si trova un capitolo dedicato al lavoro delle infermiere, scritto da Ednah Dow Cheney (1824-1904), una delle prime storiche del settore medico ${ }^{14}$.

Tra le pochissime pubblicazioni esistenti, merita di essere ricordato anche il lavoro della storica inglese Alice Clark (1874-1934), autrice del libro Working life of women in the seventeenth Century (1919), e in particolare la sezione intitolata Professions, dedicata ad infermiere, donne medico e levatrici ${ }^{15}$. Indagando nella bibliografía troviamo scarse informazioni; anche quando le donne cominciarono ad essere materia di studio, probabilmente le infermiere finirono per essere inglobate in un altro gruppo, di maggiori dimensioni ed importanza, di essere un'appendice di medici. Il riconoscimento dell'accresciuta importanza del ruolo di sanitarie, ostetriche e donne

\footnotetext{
11 Ibidem, pp. 177-186.

${ }^{12}$ MEYER NATHAN, Annie, Woman's work in America, New York, Henry Holt and Company, 1891.

13 ORTIZ GÓMEZ, Teresa, Medicina, historia y género. 130 años de investigación feminista, Oviedo, KRK, 2006.

14 ORTIZ GÓMEZ, Teresa, op. cit., pp. 89-90. Si riporta il testo dell'autrice, con particolare attenzione alle date relative alle studiose menzionate, nel caso in cui il lettore fosse interessato a conoscere la cronologia delle opere da queste realizzate.

15 ORTIZ GÓMEZ, Teresa, op. cit., pp. 94-95.
} 
medico ${ }^{16}$ può essere considerato un passo molto importante, ma non era una prassi abituale fare altrettanto con le infermiere, che non godevano dello stesso status.

Troviamo numerosi esempi dell'invisibilità del ruolo delle infermiere. Magnifiche opere sul lavoro della donna in Spagna, come quello di Mary Nash ${ }^{17}$, non possono includere tutti i lavori femminili e finiscono per limitarsi all'analisi del prototipo della donna spagnola, senza poter concentrare il campo di ricerca a lavori più specifici, come appunto quello dell'infermiera. La maggior parte delle autrici che si sono dedicate al rapporto tra donna e scienza sanno quanto ancora rimane da compiere e, nonostante nei propri libri non dedichino sezioni specifiche all'infermieristica, sono conscie di quanto sia necessario un ampio studio sulle scienze della salute e sul sistema medicoscientifico. L’opera di Teresa Ortiz e Gloria Becerra è un buon esempio dell'interesse per l'analisi, attraverso una metodologia scientifica, di alcuni dei problemi fondamentali per la storia delle donne ${ }^{18}$. A differenza di altre professioni, è inoltre singolare la scarsità di lavori che trattino la storia delle infermiere. Probabilmente si è sempre analizzata la pratica infermieristica attuale, che «continua a rispondere maggiormente ad un modello contributivo piuttosto che analitico, molto evidente nella bibliografia spagnola» ${ }^{19}$.

\section{Le infermiere spagnole tra i secoli XIX e XX}

Affinché il lavoro dell'infermiera venisse considerato una professione era necessario disporre di una serie di requisiti, in modo tale che la società lo ascrivesse a tale categoria e non continuasse a considerarlo come una mera occupazione o una professione di secondo piano. Il cammino era pieno di insidie, la prima delle quali poteva essere individuata nell'ambito educativo, dato che per possedere un titolo accademico le donne dovevano studiare, concetto quasi irrealizzabile nella Spagna tra il

16 CABRÉ, Montserrat, ORTIZ GÓMEZ, Teresa (eds.), Sanadoras, matronas y médicas en Europa. Siglos XII-XX, Barcelona, Icaria, 2001.

${ }_{17}$ NASH, Mary, Mujer, familia y trabajo en España, 1875-1936, Barcelona, Anthropos, 1983.

18 ORTIZ GÓMEZ, Teresa, BECERRA CONDE, Gloria (eds.), Mujeres de ciencias. Mujer, feminismo y ciencias naturales, experimentales y tecnológicas, Granada, Servicio de Publicaciones de la Universidad de Granada, 1996. Fin dal 2006, le due autrici hanno partecipato all'organizzazione dei Coloquios Internacionales de la Asociación Española de Investigación de Historia de las Mujeres. Il loro campo di studi ebbe risalto in un simposio dal titolo Tra salute e malattia: le donne come mediatrici del benessere; a tale studio parteciparono professionisti appartenenti a diverse discipline, provenienti da Spagna, Stati Uniti e Messico, che presentarono e discussero 15 punti relativi a vari aspetti delle pratiche sanitarie delle donne, alla loro esperienza personale diretta e alla conoscenza del corpo femminile.

19 ORTIZ GÓMEZ, Teresa, Medicina, historia y género. 130 años de investigación feminista, Oviedo, KRK, 2006, pp.198-199. 
XIX e XX secolo. Il poco peso che aveva l'educazione della donna in Spagna è evidente quando si considera la scarsa importanza assegnata all'educazione femminile: non sembrava infatti fondamentale che per lavorare fosse necessaria una formazione accademica $^{20}$. Una volta conseguita una formazione di base, si dovevano cercare scuole dove venissero insegnate le discipline relative all'infermieristica e, successivamente, era necessario cercare lavoro negli ambienti dove tale professione veniva richiesta. Il riconoscimento sociale sarebbe arrivato con il tempo, o almeno questo è quanto speravano le prime professioniste del settore. Tuttavia la realtà era ben differente, perché l'infermiera era considerata in quegli anni una sorta di medico-di-secondopiano, con tutto ciò che implicava una simile definizione. Ne conseguiva che l'infermiera guadagnasse un salario minore e che sul luogo di lavoro fosse costretta ad occuparsi di compiti dei quali un infermiere mai si sarebbe fatto carico.

$\mathrm{Al}$ giorno d'oggi, il confine tra professioni ed occupazioni è sempre meno rigido, ma agli inizi del XX secolo neppure il Diccionario de la Lengua Española presentava il termine «professionalità», categoria alla quale appunto aspiravano queste donne ${ }^{21}$. Ci sono esempi lampanti di come, non solo in Spagna, le donne cercassero di ottenere un diploma in Medicina senza riuscirci. Nel maggio 1856, Jessie Merton White chiese all'Università di Londra un permesso per iscriversi ad un corso di medicina, ma le fu negato perché venne considerata non idonea. Dopo il rifiuto, si sposò con un conte italiano e divenne la responsabile di un gruppo di donne che lavorarono come infermiere negli ospedali di Napoli ${ }^{22}$.

Fin dalla comparsa di un interesse filantropico volto a potenziare la formazione femminile nell'assistenza ai malati, il gruppo di donne che si dedicava all'infermieristica possedeva una chiara coscienza professionale, ma viveva, in realtà, una condizione differente, dal momento che il proprio lavoro, rispetto a quello dei professionisti dell'altro sesso, veniva considerato di minore qualità. La tendenza patriarcale marcava ancora i confini delle capacità e in un panorama di questo tipo le

20 I progressi raggiunti dalle donne spagnole in ambito educativo, sono menzionati in ogni studio relativo a questo settore. In questo articolo si accennerà ad una delle autrici che maggiormente hanno approfondito tale ambito, senza per questo voler sminuire il merito dei molteplici studi portati a termine sull'argomento, CAPEL MARTÍNEZ, Rosa María, El trabajo y la educación de la mujer en España (1900-1930), Madrid, Ministero di Cultura, 1982. Fino alla Costituzione del 1869, in Spagna non era riconosciuto il diritto all'educazione, CAPEL MARTÍNEZ, Rosa María, op. cit., pp. 176-177.

${ }^{21}$ La distinzione tra «professioni classiche» e «sottoprofessioni» è presente fin da quando Max Weber definì la professione come «l'attività specializzata e permanente di un uomo, che, normalmente, costituisce per lui una fonte di reddito e, di conseguenza, una sicura base economica della sua esistenza», cit. in CORTINA, Adela, «Profesionalidad» in CEREZO GALÁN, Pedro (ed.), Democracia y virtudes cívicas, Madrid, Biblioteca Nueva, 2005, pp. 361-381.

${ }^{22}$ SÁNCHEZ RON, José Manuel, El poder de la ciencia. Historia social, política y económica de la ciencia (siglos XIX y XX), Barcelona, Crítica, 2007, p. 267. 
donne non potevano aspirare al riconoscimento che meritavano. Tale disuguaglianza è evidente fin dagli albori, o per lo meno fin dall'epoca medievale, dato che esistono documenti del 1316 nei quali si usa la parola infermiere per indicare i compiti nell'assistenza agli infermi, mentre la definizione di infermiera non compare quasi mai in modo esplicito, nonostante spesso fossero proprio le donne a doversene incaricare ${ }^{23}$. Questa realtà conferma le difficoltà che le donne avrebbero incontrato nel trovare uno sbocco professionale. Alcuni autori furono molto chiari nel sostenere quali fossero le differenze tra tirocinanti ed infermiere. Nel 1923, il medico Emilio Alonso y García Sierra affermava che:

[...] Esiste un'enorme differenza tra un tirocinante ed un infermiere ed è doveroso evidenziarla perché ognuno possa far valere i propri diritti. Il Tirocinante è colui che possiede un titolo professionale, che, seppur inerente ad un ambito più limitato, gode dello stesso rispetto di ogni altro tipo di corso di studi ufficiale ed esercita le prescrizioni del medico, inerenti alla scienza. L’infermiere è una qualsiasi persona che esegue gli ordini del medico, mettendoli in pratica [...] ${ }^{24}$.

È stato inoltre dimostrato che le infermiere sono sempre state considerate come lavoratrici di livello inferiore. Jesús de Miguel sostiene che: «si continua a considerare l'infermiera come un medico-di-secondo-piano, inferiore e con conoscenze similari, ma di minor livello, e non come un profilo distinto, unico ed autonomo» 25 . Con queste premesse, si evince che quella dell'infermiera non era una attività riconosciuta dalla società; prova ulteriore ne è il fatto che la parola infermiera non apparve in documenti ufficiali fino all'anno 1756, quando oltretutto venne usata per riferirsi alla responsabile di un gruppo di infermiere di un convento ${ }^{26}$. Alla luce di questo dato, è facile intuire come la discriminazione dipendesse dal sesso, o dallo stretto rapporto con la Chiesa e le iniziative umanitarie e caritatevoli, considerate più consone alla sfera femminile.

23 DOMÍNGUEZ-ALCÓN, Carmen, Los cuidados y la profesión de enfermera en España, Madrid, Pirámide, 1983, p. 62.

24HERNÁNDEZ MARTÍNEZ, Francisca, PINAR GARCÍA, María Eugenia, MORENO ROY, María Ángeles, «Memoria de un centenario. Primera Escuela de Enfermería Santa Isabel de Hungría», in Híades. Revista de Historia de la Enfermería, 3-4/1996-1997, p. 194. Era ritenuto fondamentale che tutti i profili sanitari fossero al corrente di tale distinzione, per sapere quale fosse il proprio compito nell'ambiente in cui si muovevano, sempre sotto la tutela del medico. ${ }_{25}$ In diverse occasioni le infermiere vengono considerate come «medici-di-secondo-piano, inferiori, con conoscenze simili ma minori», cit. in MIGUEL, Jesús M. de, El mito de la inmaculada concepción, Barcelona, Anagrama, 1979, p. 128. Si tratta di uno degli autori ricordati nelle menzioni bibliografiche del libro di ORTIZ GÓMEZ, Teresa, Medicina, historia y género, cit., pp. 118, 285.

${ }^{26}$ DOMÍNGUEZ-ALCÓN, Carmen, Los cuidados y la profesión de enfermera en España, cit., p. 72. 
Per ciò che riguarda i paesi di tradizione cattolica, come appunto la Spagna, non si può dimenticare la relazione tra l'infermieristica e le religiose sanitarie che si dedicavano alla cura del malati, questione che ha dato luogo a diverse errate interpretazioni. Da un lato, un gruppo di studiosi strettamente legato alla professione delle infermiere, si mostra critico rispetto all'importanza attribuita all'orientamento vocazionale delle infermiere religiose, sostenendo che poteva rappresentare un freno al loro pieno riconoscimento come professioniste del settore. Alcune studiose collaboratrici del progetto Híades, invece, hanno cercato di dimostrare come il vincolo tra suore ed infermieristica influisse positivamente e non implicasse un allontanamento dalla professionalità ${ }^{27}$. In un articolo sulla Congregazione delle Figlie della Carità, viene esposta l'importanza di tale congregazione al momento della sua fondazione, da parte di San Vicente de Paul e Santa Luisa de Marillac, nella prima metà del XVII secolo, in Francia: invece di allontanarsi dalla loro professione, il gruppo denominato Figlie della Carità fu capace di far fronte alla cura degli ammalati in Spagna, insediandosi nella maggior parte dei centri di beneficenza pubblica. Il suo ingresso in Spagna risale alla fine del XVIII secolo, grazie ad un accordo del governo spagnolo con il Conte di Floridablanca, che ne permise l'insediamento nel paese ${ }^{28}$. All'inizio, nel 1790, si stabilirono a Barcellona (Hospital de la Santa Cruz), per passare successivamente a Lerida, a Reus, e arrivare a Madrid (Hospital de la Inclusa), nel 1800. In questi centri, la presenza delle suore infermiere diede luogo ad alcuni problemi, per la maggior parte causati dal personale dipendente degli ospedali, che vedeva in queste una concorrenza sleale per motivi economici. La loro opera ebbe un peso così rilevante da giungere a promuovere l'organizzazione dell'assistenza agli ammalati in gran parte della Spagna, inserendosi, a partire dall'anno 1915, nel processo di professionalizzazione del settore. La tesi volta ad evidenziare i benefici ottenuti, invita ad un confronto tra gli studi compiuti dalle infermiere della Congregazione delle Serve di Maria e quelli della Facoltà di Medicina di Madrid: la vocazione non rappresentò in nessun momento un freno nel percorso verso la professionalità e già dal 1915 sembra che fosse in atto un progetto di realizzazione di un Manuale sulla cura degli ammalati.

Nel 1917 si pubblicò la prima edizione del libro Manual de la Carrera de Enfermeras para uso de las Hijas de la Caridad Españolas, chiaro esempio del rigore con cui esse organizzavano le proprie attività. L'articolo dimostra come la vocazione non avesse costituito un ostacolo: per dar spazio a questa tesi sono stati confrontati

${ }^{27}$ HERNÁNDEZ MARTÍN, Francisca, MORENO ROY, María Ángeles, PINAR GARCÍA, María Eugenia, «De las Hijas de la Caridad y su formación», in Híades. Revista de Historia de la Enfermería, 2, 1995, pp. 169-177.

28 Ibidem, p. 175 . 
diversi manuali, che permettono di dimostrare il valore delle prime religiose promosse infermiere. Vengono chiaramente spiegati i presupposti sulla base dei quali è stato operato un confronto tra i manuali dell'epoca e come il manuale coincidesse con il programma ufficiale degli studi di infermiera stabilito dal governo stesso. Nonostante sia inevitabile riconoscere l'importanza degli ordini religiosi ed il loro rapporto con l'infermieristica, non possiamo dimenticare che in Spagna, come del resto in Europa, dove a causa della partecipazione alle due Guerre Mondiali si doveva contare su servizi sanitari capaci di porre rimedio alle situazioni tragiche prodotte da un evento bellico, si produssero vari tentativi di potenziamento della formazione del personale laico nell'ambito sanitario.

Le prime scuole laiche per infermiere comparvero in Spagna nel XIX secolo, per iniziativa dello Stato. Fin dall'inizio i decreti cercarono di porre ordine nell'accesso alle professioni di ostetriche, infermiere e tirocinanti, dove le prime infermiere cominciavano a muovere i loro primi passi. Come indica Teresa Ortiz, verso la fine del XIX secolo si cercò di far nascere un nuovo corso di studi, diretto esclusivamente alle donne, l'infermieristica:

l'unico esempio di infermieristica laica di base, tecnica e formalmente regolata, era costituito in Spagna dal gruppo dei tirocinanti, creato nel 1857 e, precedentemente, (1846-1856) da quello dei praticanti. Entrambe erano professioni riservate esclusivamente agli uomini29.

Appena comparvero le prime scuole, si stabilirono programmi di studio per le donne, che si sarebbero dedicate alla cura dei malati. Tuttavia, fin dall'inizio, questa attività celava un'insidia, dato che, nella maggioranza dei casi, le infermiere dipendevano dalle decisioni prese da altri profili professionali, sempre legati alla sanità, ma che occupavano posizioni più elevate. Proprio questo interesse, ancora inesistente in Spagna, propiziò la necessità di creare scuole per infermiere, che potessero rendere le donne capaci di svolgere una professione fondamentale durante le guerre ${ }^{30}$. Nonostante il rapporto delle infermiere spagnole con la Guerra Civile del 1936 sia stato oggetto di numerosi articoli che ne analizzano aspetti concreti, non esistono opere che trattino il ruolo delle infermiere durante tale conflitto. Conosciamo alcuni dati relativi

29 ORTIZ GÓMEZ, Teresa, «Profesiones sanitarias», in MORANT, Isabel (dir.), Historia de las mujeres en España y América Latina, Vol. III, Madrid, Cátedra, 2006, p. 529.

30 «Le professioni classiche apportano allo stesso tempo sia il sapere esclusivo di un settore in concreto, che l'impegno a raggiungere determinate mete». Si veda, CORTINA, Adela, «Profesionalidad», in CEREZO GALÁN, Pedro (ed.), op. cit., p. 363. 
alla partecipazione della Croce Rossa alla guerra, riguardo la loro assistenza super partes alle vittime del conflitto, tanto del lato repubblicano come del nazionalista, ma non sono tuttavia stati scritti libri che affrontino l'argomento in modo approfondito ${ }^{31}$.

\section{Le leggi fondamentali}

Affinché la situazione cambiasse, si cercarono di unire il filantropismo e alcune misure di carattere politico, nell'intento di migliorare la penosa situazione in cui versava l'assistenza sanitaria spagnola. Nel 1827 medici e chirurghi furono inseriti in un'unico percorso di studi universitario, nel tentativo di riorganizzare entrambi i corsi; tuttavia, nel 1845 esistevano ancora modi differenti per definire le diverse professioni riconducibili alla professione di medico, come quella del chirurgo "romancista", che veniva ora definito "praticante" e che si dedicava a realizzare operazioni minori che non era solito realizzare il medico. Il chirurgo "romancista", secondo il dizionario, era il chirurgo che non conosceva il latino, mentre il "praticante" era il chirurgo che realizzava operazioni di minor importanza.

Il cambio più importante si produsse nel settembre del 1857, con l'approvazione della "Legge fondamentali dell'Istituzione Pubblica", altresì denominata Ley Moyano, che pose le basi per lo sviluppo e il miglioramento delle professioni sanitarie. Questa legge rappresentò un punto fondamentale per la situazione sanitaria spagnola, dato che poco dopo venne pubblicato un "Regolamento per la formazione del tirocinante e della levatrice", che diede vita al corso di tirocinante, al quale vennero attribuite tre distinte specializzazioni: callista, dentista ed assistente ai parti. Come spesso succedeva, le norme fissate per risolvere i problemi sanitari risultarono discriminatorie e fin dall'inizio le donne rimasero emarginate. Prima di raggiungere il diritto al lavoro, dovettero lottare per ottenere un diritto elementare, cioè quello all'educazione ed alla formazione, ambiti ai quali veniva loro proibito l'accesso32.

\footnotetext{
${ }^{31}$ SILES GONZÁLEZ, José, «Enfermería y conflictos bélicos: una historia por hacer», in Índex de Enfermería, 15, 1996, pp. 7-8. Tale titolo è chiaro esempio della carenza di opere che trattino infermieristica e guerra civile spagnola.

$3^{2}$ Alle donne fu negato, oltre all'opportunità di formarsi, la possibilità di integrarsi nella società una volta ricevuta la formazione. Come afferma Isabel Cabrera Bosch, «secondo il Regio Decreto del luglio 1878 , le donne potevano ottenere il titolo di scuola media superiore, solo a condizione che questo non venisse usato per esercitare una professione», cit. in CABRERA BOSCH, Isabel, «Ciudadanía y género en el liberalismo decimonónico español», in PÉREZ CANTÓ, Pilar (ed.), También somos ciudadanas, Madrid, Instituto Universitario de Estudios de la Mujer, Universidad Autónoma de Madrid, 2004, p. 201.
} 
Nel XIX secolo, alcune donne legate al Krausismo33 ed all'istituzione libera dell'insegnamento, difesero l'idea che fosse necessario modernizzare la vita delle donne e che venisse loro permesso dedicarsi all'infermieristica, attività che appariva più vicina ai valori femminili. Concepción Arenal non fu l'unica che rifletté sul bisogno di educare le donne perché avessero la possibilità di professionalizzarsi34. La storica spagnola Rosa María Capel ha commentato i dibattiti che si produssero sull'argomento, verso la fine del secolo XIX. Il "Congreso Nacional Pedagógico", che si svolse a Madrid il 17 maggio 1882, ci fornisce infatti gli strumenti per comprendere il ritardo educativo della Spagna35. Secondo quanto venne esposto durante i dibattiti del Congresso, era necessario che si producessero miglioramenti nell'esercizio professionale; venne affermato che le donne potevano accedere unicamente a determinati studi finalizzati a intraprendere poche professioni, nel campo dell'«Educazione di base e primaria, Belle Arti e Musica; Lavori d'ufficio; Poste e comunicazioni; Farmacia; Medicina nelle specializzazioni femminili; Industrie» ${ }^{36}$. Alle donne era inoltre permesso partecipare pienamente a queste attività, ma era ritenuta inadeguata per ogni altro ambito. In questo lento cammino verso una maggior uguaglianza, il $\mathrm{XX}$ secolo cominciò sotto buoni auspici, dal momento che, oltre a ridursi il tasso di analfabetismo, quindici donne avevano terminato con successo i propri studi universitari37.

\footnotetext{
33 Il Krausismo è una dottrina che difende la tolleranza accademica e la libertà d'insegnamento, in opposizione al dogmatismo. Si tratta di un movimento filosofico che ebbe una forte ripercussione in Spagna, dove raggiunse la sua massima diffusione grazie alle opere del suo principale divulgartore, Julián Sanz del Río, e all'Istituzione Libera dell'Insegnamento diretta da Francisco Giner de los Ríos, oltre al contributo dato dal grande giurista Federico de Castro. Deve il proprio nome al pensatore postkantiano tedesco Karl Christian Friedrich Krause (17811832).

34 ARENAL, Concepción, La emancipación de la mujer en España, cit., p. 36.

35 CAPEL MARTíNEZ, Rosa María, «La apertura del horizonte cultural femenino: Fernando de Castro y los congresos pedagógicos del siglo XIX», in ID. (ed.), Mujer y Sociedad en España (1700-1975), Madrid, Ministerio de Cultura, Dirección General de Juventud y Promoción SocioCultural, 1982, pp. 109-145.

${ }^{36}$ Ibidem, p. 141.

37 Rosa María Capel ci fornisce alcuni dati provenienti dal suo studio sulla Contessa di Campolange, nel libro CAPEL MARTíNEZ, Rosa María, La mujer en España. Cien años de su historia, Madrid, Aguilar, 1964, pp. 166-168. Si veda anche: CAPEL MARTÍNEZ, Rosa María, «La apertura del horizonte cultural femenino: Fernando de Castro y los congresos pedagógicos del siglo XIX», in ID. (ed.), Mujer y Sociedad en España (170o-1975), cit., p. 144.
} 


\section{Le scuole di infermieristica: un avvicinamento alla professionalizzazione}

Sebbene nella maggioranza dei paesi occidentali le prime scuole per infermiere sorsero a partire dalla metà del XIX secolo, in Spagna ciò non accadde fino al XX secolo $^{38}$. Fu Florence Nightingale chi influì maggiormente nella creazione di Scuole per Infermiere in Europa, tra le quali una delle più conosciute era la Scuola di San Tommaso. La pubblicazione del suo libro "Notas sobre enfermería" indicò le linee guida per dare risalto alla professione. Nonostante ciò, i primi tentativi di creare centri per la cura degli infermi che potessero rendere la Spagna simile ad altri paesi europei come Inghilterra, Austria, Francia o Germania, risalivano a un periodo precedente. Nel 1858, Pedro Felipe Monlau pubblicò un articolo per la rivista «El Monitor de la Salud de las Familias», dal titolo «L'arte di assistere gli ammalati», nel quale si accennava al bisogno di creare scuole per infermieri ed infermiere ${ }^{39}$. Sarebbe stato però necessario attendere ancora molto tempo.

Verso la fine del XIX secolo, nell'ottobre del 1896 secondo la maggior parte degli studiosi, ${ }^{40}$ si creò presso l'Instituto Rubio y Galí la Escuela de Enfermeras Santa Isabel de Hungría, ovvero la prima scuola per infermiere di Spagna, fondata dal Dottor Federico Rubio y Galí, sfruttando gli edifici dell'antico Instituto Operatorio Federico Rubio y Galí. Affinchè le infermiere ricevessero un'educazione regolare, era necessario porre le basi che avrebbero sostenuto i loro studi di infermieristica. Tuttavia non abbiamo conferma dell'esistenza di un percorso per tali studi fino all'anno 1915, grazie alla pubblicazione di un Real Orden, un regio decreto, con il quale si riconobbe l'esistenza di un programma ufficiale per l'insegnamento della professione dell'Infermieristica ${ }^{41}$. Il ritardo delle scuole di infermieristica spagnole rispetto a quelle

$3^{8}$ ÁLVAREZ RICART, M. del Carmen, La mujer como profesional de la medicina en la España del siglo XIX, Barcelona, Anthropos, 1988.

39 In Europa, in questi anni, si fondarono scuole per infermiere.

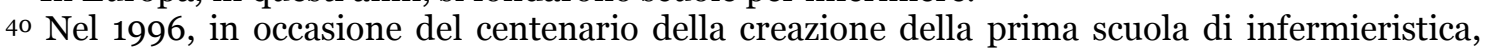
furono pubblicati alcuni articoli. La rivista Híades ricorda come la sua istituzione fu opera del dottore Federico Rubio y Galí, Ambasciatore di Spagna in Inghilterra durante il decennio 18601870. Dato che all'Ambasciatore risultava complicato imitare il modello inglese, decise di creare, presso l'Instituto Operatorio Federico Rubio y Galí, la prima scuola per infermiere di Spagna. Si veda HERNÁNDEZ MARTÍNEZ, Francisca, PINAR GARCÍA, María Eugenia, MORENO ROY, María Ángeles, cit., pp. 189-195.

${ }^{41}$ Ibidem, p. 194. Le infermiere che si dedicavano all'assistenza, avevano bisogno di una preparazione che andasse al di là della semplice applicazione di cataplasmi; da qui il testo normativo (R.O. del 7 di maggio, pubblicato il 21 Maggio 1915). 
in Inghilterra o Francia, fu una diretta conseguenza della tarda istituzionalizzazione dell'igiene e della salute pubblica ${ }^{42}$.

Alla Escuela de Enfermeras Santa Isabel ne seguirono altre, come per esempio quella della Santa Madrona a Barcellona, fondata dal Montepío de la Santa Madrona nell'aprile del 1917, che non nascose mai la propria intenzione di promuovere il ruolo della donna43. In queste prime scuole ci troviamo di fronte ad un contraddizione, in quanto erano sì per infermiere, ma le lezioni venivano impartite da medici di sesso maschile; allo stesso modo, i primi manuali furono scritti da uomini. Nella Escuela de la Santa Madrona di Barcellona, solo il corso di studi in "Anatomia e Fisiologia" era impartito da un medico donna44. Sempre nel 1917, e sempre a Barcellona, venne fondata la Escuela de Enfermers Auxiliares de Medicina de la Mancomunidad de Catalunya. Ciò che più ci interessa di questa scuola è che, anche se fondata nel 1917, non iniziò a funzionare che qualche anno più tardi, vale a dire nel 1922, in cui la stessa scuola emanasse un documento nel quale si auspicava il miglioramento della posizione della donna nell'ambito infermieristico:

Si apre un nuovo e nobilissimo campo per l'attività femminile, che oltre a contribuire in modo positivo al miglioramento sociale e culturale della donna catalana, può rappresentare un potente mezzo per la diminuzione delle malattie $\mathrm{e}$ della mortalità nella nostra terra45.

Dobbiamo evidenziare come la direzione della nuova scuola catalana si trovasse in mano ad un patronato formato da medici di sesso maschile, ma che contava anche sulla presenza di due donne, questione che, rispetto a precedenti patronati formati unicamente da personale maschile, rappresentava un importante passo in avanti. Tra i professori figuravano inoltre medici uomini, un sacerdote e due professoresse ${ }^{46}$. Sembra che la scuola fosse caratterizzata da un carattere altamente progressista, peculiarità che le permise di distinguersi da altre esistenti in Spagna nello stesso

42 BERNABEU MESTRE, Josep, GASCÓN PÉREZ, Encarna, Historia de la Enfermería de Salud Pública en España (1860-1977), Murcia, Universidad de Alicante, 1999, p. 20.

43 Questa scuola è tra quelle che maggiormente sostennero la professionalizzazione dell'infermieristica. Cfr. HERRERA RODRÍGUEZ, Francisco, «La enseñanza de las Damas Enfermeras de la Cruz Roja (1917-1920)», in Híades, Revista de Historia de la Enfermería, 3-4, 1996-1997, p. 198.

${ }^{44}$ La persona in questione era Carmen Díaz, un esempio per le infermiere assegnate ai turni di notte; questo sottogruppo riceveva un'istruzione teorica più ridotta rispetto ad altre infermiere. Si veda DOMÍNGUEZ-ALCÓN, Carmen, op. cit., p.114.

45 Ibidem, p.117.

${ }^{4}$ Secondo quanto indicato da Carmen Domínguez-Alcón, si trattava di Ángeles Bosch de Esquerdo e Ángeles Mateu. Ibidem. 
periodo. Ma si continuò a fondarne altre: nel 1926 la Escuela de Enfermeras de la Quinta de Salud la Alianza, e nel 1933 L'Ecola d'Infermeres de la Generalitat de Cataluña, inserita all'interno di un piano catalano di miglioramento culturale, sanitario ed igienico. Fin dalla sua fondazione, l'obiettivo prioritario de L'Ecola d'Infermeres de la Generalitat de Cataluña era quello di conseguire una formazione di qualità per il personale preposto a fornire servizi ospedalieri. Difatti, una delle sue finalità era offrire un mezzo di vita nobile, degno e remunerato a giovani donne che si distinguessero per l'«alto grado di spiritualità e altruismo»47. Il carattere aperto e all'avanguardia si doveva alla donna incaricata della sua gestione, Ripol Noble, una professionista "moderna". Formatasi come infermiera negli Stati Uniti grazie ad una borsa di studio della fondazione Rockefeller, la sua preparazione la portò a diventare la responsabile delle indagini del centro. Contribuí inoltre a far sì che la Scuola non si dedicasse solo all'insegnamento di materie specifiche inerenti la cura degli ammalati, ma perseguisse anche la formazione culturale, programmando concerti, opere, conferenze, ed un insieme di attività differenti, finalizzate alla formazione integrale dell'individuo. Anche la Croce Rossa ebbe un ruolo importante nel processo di professionalizzazione delle infermiere. Nel 1924 venne pubblicato il Manual de la Dama Efermera de la Cruz Roja Española ${ }^{48}$, che rappresentò la base per la realizzazione di un programma di scuole per infermiere in tutto il paese. La forza del Corpo fu tale, che nel 1929 l'insegnamento ad infermiere era già istituito in trentadue province spagnole.

L'opera della Croce Rossa non contribuì solamente alla professionalizzazione delle infermiere. In molte città spagnole convivevano zone di livello signorile con altre emarginate, dove non erano presenti i servizi necessari, e le condizioni di vita erano insufficienti per mantenere integra la salute degli abitanti di quei quartieri. L'esistenza della Scuole per Infermiere propiziò la creazione di numerosi ospedali che conferirono ad alcune città spagnole un aspetto moderno e salubre. Croce Rossa Spagnola, con l'appoggio del governo, inaugurò nel 1918 i suoi primi sei ospedali a Madrid, Bilbao, Barcellona, Granada, San Sebastian e Ceuta, mentre nell'ospedale di San José y Santa Adela di Madrid fu fondata la prima Scuola per Infermiere di Spagna 49.

\footnotetext{
47 Ibidem.

${ }^{48}$ Tra varie fonti, si citano le seguenti opere: FERNÁNDEZ-CORREDOR Y CHICOTE, Mariano, Manual de la enseñanza de Enfermeras, Valladolid, Talleres Tipográficos "CUESTA", 1917; MARTÍNEZ VARGAS, Andrés, "Manual para las Damas Enfermeras de la Cruz Roja», in SITGES CREUS, Antonio (ed.), Manual de enfermería, Barcelona, Torray, 1966, anexo 8 (1917), anexo 9 (1918).

49 Cfr. CLEMENTE, Josep Carles, La Escuela Universitaria de Enfermeras de Madrid, cit., p. 32.
} 
Tuttavia, si viveva una situazione contraddittoria e le scuole per infermiere non sempre erano sinonimo di buona formazione. Riguardo al rapporto tra l'insegnamento teorico e la sua messa in pratica, è opportuno citare un aspetto concreto: nel 1929 la Società Spagnola di Igiene attribuì un premio a Nieves González Barrio5o, per avere scritto un libro sulla formazione delle infermiere per l'attività di visita. Tale scritto era meritevole di numerosi elogi perché spiegava molto chiaramente quale fosse la teoria del lavoro delle infermiere di visita, nonostante allo stesso tempo lamentasse insistentemente una scarsa preparazione pratica delle infermiere stesse ${ }^{51}$. L'autrice poneva una serie di questioni sull'infermieristica, sviluppate grazie a uno studio effettuato sulle infermiere durante la loro attività di visita, e sulla difesa delle scuole di carattere pubblico affinché venisse loro riconosciuta la dignità accademica, posizione di cui già godevano altre professioni sanitarie. Giunse addirittura ad affermare che «un'infermiera incompetente poteva essere pericolosa quanto un medicastro» 52 .

Oltre all'enorme impulso verso la professionalizzazione, grazie all'ingente opera fondatrice di ospedali e scuole della Croce Rossa, è doveroso ricordare il ruolo che ebbe in Spagna la Regina María Cristina di Asburgo, seconda moglie di Alfonso XII e madre di Alfonso XIII, che esercitò il ruolo di reggente negli anni intercorsi tra la morte del primo e l'ascesa al trono del secondo53. Se la persona incaricata di organizzare l'opera di sostegno ed aiuto ai poveri, ai sofferenti ed ai malati era una regina, tutto cambiava radicalmente. Grazie al doppio ruolo di Regina Reggente e Regina Madre, favorì la costruzione del Sanatorio Central de Vallehermoso a Madrid, inizialmente destinato alla cura degli operai rimasti feriti sul luogo di lavoro. In seguito appoggiò la costruzione di altri ospedali a San Sebastian e Irún, sanatori dove sarebbero arrivati i feriti della guerra di Cuba. La Regina María Cristina non fece altro che promuovere l'opera della Croce Rossa Spagnola, che aveva iniziato a costruire ospedali nel 1872 , consolidando in questo modo un aspetto fondamentale per un paese impegnato in diversi conflitti bellici.

50 GONZÁLEZ BARRIO, Nieves, Organización y reglamentación del cuerpo e institución de enfermeras visitadoras y acción cultural de las mismas, Madrid, Sociedad Española de Higiene, 1930. Il lavoro premiato, uno dei contributi della Dott.ssa González Barrio al sistema medico spagnolo, si ispirava alla sua esperienza negli USA, e più in concreto alla relazione della Rockfeller Foundation e la National Organization for Public Health Nursing. Vid., BERNABEU MESTRE, Josep, GASCÓN PÉREZ, Encarna, Historia de la Enfermería de Salud Pública en España (1860-1977), Murcia, Publicaciones de la Universidad de Alicante, 1999, p. 51 et seq.

${ }^{51}$ DOMÍNGUEZ-ALCÓN, Carmen, Los cuidados y la profesión de enfermera en España, op. cit., p. 118.

$5^{2}$ BERNABEU MESTRE, Josep, GASCÓN PÉREZ, Encarna, op. cit., pp. 42.

53 CLEMENTE, Josep Carles, Tiempo de humanidad. La labor sanitaria de la Cruz Roja

Española (1864-1997), Madrid, Fundamentos, 2004, pp. 9, 13. 
La Croce Rossa ricevette sempre l'appoggio della Famiglia Reale, ma la sua fondazione fu dovuta al Conte di Ripalda. Grazie al lavoro di Josep Carles Clemente54, sappiamo che il primo nucleo della Croce Rossa in Spagna risale al 2 marzo 1864, quando ancora non era conosciuta come Croce Rossa, ma come Asociación Internacional de Ayuda a los Heridos de Guerra de Mar y Tierra. Sección española. I passi successivi per la sua formazione furono possibili grazie all'iniziativa del fondatore della Croce Rossa, Jean Henry Dunant, che si incaricò di richiedere all'Ordine di San Giovanni di Gerusalemme l'invio di una rappresentanza spagnola ad una conferenza internazionale che si sarebbe celebrata a Ginevra, dal 26 al 29 ottobre 1863. L'Ordine di San Giovanni di Gerusalemme di Spagna mandò Joaquín de Agulló, Conte di Ripalda, e il Ministero della Guerra inviò Nicasio Landa, medico superiore della Sanidad Militar. Entrambi i rappresentanti spagnoli si impegnarono a creare una società nazionale volta alla protezione, in tempo di guerra, dei feriti causati dai conflitti. Si trattava di un programma filantropico che richiedeva la collaborazione delle autorità dei paesi in guerra affinchè facilitassero l'opera di soccorso. I membri dell'associazione avrebbero indossato un'uniforme ed un segno distintivo per essere identificati, una croce rossa su fondo bianco: gli infermieri e le infermiere volontari avrebbero dovuto essere persone preparate per aiutare e soccorrere i soldati feriti. Questa era la base della creazione di un Corpo di infermiere, che avrebbe assunto un ruolo di grande rilevanza. L’informazione piú approfondita riguardo alla creazione della Croce Rossa in Spagna si trova nel libro già citato; non affronteremo qui il percorso della sua creazione, bensì ci dedicheremo ad analizzare come vennero promossi diversi progetti per modificare l'atteggiamento oscurantista proprio della società spagnola. Fin dal 1864, anno della sua fondazione, la Croce Rossa conobbe diverse fasi, una delle quali viene denominata "Victoria Eugenia" e comprende gli anni dal 1916 al 1924. La società spagnola di questi anni era profondamente segnata dal conservatorismo ideologico, insieme ad un distanziamento da ogni pratica sanitaria, motivo per cui l'impulso dato alla Croce Rossa produsse per il popolo diversi benefici a livello sanitario. La monarchia stessa collaborò con la Croce Rossa, nominando la regina Vittoria Eugenia autorità suprema in tempi di guerra55.

\footnotetext{
54 Ibidem.

55 HIJANO PÉREZ, Maria Ángeles, Victoria Eugenia de Battenberg. Una reina exiliada, Madrid, Alderabán, 2000, pp. 152-153.
} 


\section{La spinta della monarchia e la sua collaborazione con la Croce Rossa}

Oltre all'opera filantropica di María Cristina, dobbiamo ricordare anche quella della Regina Victoria Eugenia, moglie di Alfonso XIII, che comprende gli anni dal 1916 al 1924. La Rregina assunse una posizione molto vicina alla Croce Rossa: il momento più importante coincise con la riorganizzazione della Sezione Spagnola della Croce Rossa, attraverso il Decreto Regio del 16 gennaio 1916, anche se gli statuti della nuova sezione non furono fissati fino al 16 giugno dell'anno successivo. In tale decreto, si affermava espressamente che Sua Maestà il Re avrebbe assunto il mandato supremo sulla Croce Rossa, riservandosi la possibilità di delegarlo alla Regina, che l'avrebbe sempre assunto in periodo di guerra. A partire da tale decreto, tutti i documenti relativi alla Sezione Spagnola della Croce Rossa indicano la sua dipendenza diretta dal Ministero della Guerra e da quello dell'Esercito per questioni militari, e dal Ministero degli Esteri per questioni internazionali. Da questo momento, la Regina sarebbe diventata la Presidentessa dell'Assemblea Suprema della Croce Rossa ${ }^{6}$. Gli archivi della Croce Rossa di Madrid possono fornire informazioni sul ruolo della Regina, e nonostante si tratti di un’informazione poco chiara e discontinua, può comunque apportare qualche dato sull'attività principale dei suoi ultimi anni come Regina di Spagna57. La sua partecipazione come autorità suprema della Croce Rossa non fu sufficiente per guadagnare la stima degli Spagnoli. Questo atto di magnanimità era fondamentale per la Regina e per buona parte dell'alta società spagnola, ma c'era bisogno di qualcosa in più. Victoria Eugenia pensò che grazie alla posizione di cui godeva nella Croce Rossa, avrebbe potuto fare qualcosa per guadagnare l'affetto dei cittadini, e decise quindi di

${ }^{56} \mathrm{Nel}$ decreto si indicavano sette punti per riorganizzare la Sezione Spagnola della Croce Rossa, ma l'aspetto che più ci interessa è quello inerente Victoria Eugenia; veniva infatti affermato come l'autorità suprema della Croce Rossa spettasse alla Regina di Spagna, sia nell'eventualità in cui il Re avesse deciso spontaneamente di attribuirlo a lei, sia in caso di guerra. Inoltre veniva incaricata della presidenza delle cosiddette Secciones de Señoras e, fondamentalmente, del controllo dell'Istituzione che lei stessa aveva seguito. Lo stesso decreto, firmato da Alfonso XIII, stabiliva alcuni parametri per evitare l'ingente spesa che avrebbe comportato un'organizzazione di tali dimensioni. In questo modo, si decise che la Ley de Presupuestos del Estado avrebbe sovvenzionato la nuova istituzione con 50.000 pesetas, cifra che fu poi investita in Borsa tramite obbligazioni del Tesoro. Contemporaneamente ne vennero nominate tutte le alte cariche: ispettore, contabile, tesoriere e segretario generale. Victoria Eugenia rimase molto soddisfatta, in quanto le spettava il privilegio di nominare il rappresentante dell'Assemblea Centrale delle Donne.

${ }_{57}$ A.C.R., Revista Cruz Roja, 213, marzo 1920; A.C.R., Revista Cruz Roja, 214, maggio 1920. 
sfruttare i propri contatti per formare un Corpo di Infermiere della Croce Rossa spagnola.

La richiesta giunse a tutti i livelli che dovevano incaricarsi dell'elaborazione ed approvazione del suo regolamento, fino al Ministero della Guerra, e da lì alla sezione di giustizia per l'approvazione definitiva, il 18 maggio 1917. Il regolamento constava di quattro capitoli, nei quali si spiegavano i requisiti necessari per poter entrare a far parte del Corpo. Nel primo capitolo, il primo articolo dichiarava che la Regina era la responsabile superiore del Corpo, mentre il secondo indicava gli incaricati della direzione e del governo del Corpo stesso. I successivi capitoli, invece, regolavano i corsi, gli esami e l'entrata nel Corpo (II), le categorie e le corrispettive funzioni (III), le uniformi, l'emblema e la fascia (IV). La creazione di questo Corpo di Donne Infermiere avrebbe permesso ad un'ampia parte della popolazione femminile di studiare per ottenere il titolo di infermiera o per svolgere lavori alle dipendenze delle strutture create per l'apprendimento. La popolazione rimase molto soddisfatta, in quanto si creavano i presupposti perchè gli ammalati ricevessero un miglior trattamento e si producessero nuovi posti di lavoro. La sua opera non finì qua, dato che si batté perché il Regolamento delle Infermiere consentisse l'accesso, previo esame teorico e pratico negli ospedali, di tutte le religiose appartenenti a qualsiasi ordine legalmente riconosciuto in Spagna. L'ampliamento fu riconosciuto dal Ministero della Guerra il 7 febbraio 1918 e Victoria Eugenia si ingraziò in questo modo parte degli ecclesiastici. L'importanza di tale atto fu notevole e venne seguita dalla pubblicazione, sulla rivista mensile del Corpo di infermiere della Croce Rossa, di diversi articoli che descrivevano i benefici che ne sarebbero scaturiti. Il numero 213 della rivista, ad esempio, pubblicato nel marzo del 1920, presentava un articolo sulla questione ${ }^{58}$.

Sull'onda degli elogi e dell'entusiasmo, Victoria Eugenia accelerò la sua missione, iniziata il 22 gennaio 1918, con la creazione del Hospital Central di Madrid, inaugurato ufficialmente il 23 dicembre 1928, come Hospital de San José y Santa Adela. Questo ospedale fu donato alla Croce Rossa come sede per la scuola per infermiere59. A quei tempi, aveva una capienza di 72 letti e tutte le attrezzature più moderne, oltre a poter offrire alle alunne la possibilità di risiedervi. Presto la popolazione spagnola si sarebbe resa conto di come quell'impiego, poco conosciuto fino ad allora, potesse rappresentare il mezzo di sussistenza per molte donne ${ }^{60}$. Fu fondamentale un'unione di intenti, tra cui

${ }^{8}$ HERRERA RODRIGUEZ, Francisco, «La enseñanza de las Damas Enfermeras de la Cruz Roja (1917-1920)», in Híades, 3, 1996, pp. 197-209.

59 Cfr. CLEMENTE, Josep Carles, Tiempo de humanidad, cit., p. 15.

6o Per maggiori informazioni sul ruolo di Victoria Eugenia si veda HIJANO PÉREZ, Ángeles, Victoria Eugenia de Battenberg, Madrid, Alderabán Ediciones, 2000, pp. 175-180. 
quelli di alcuni nobili come il Conte di Ripalda, per creare un fondo di risorse capace di far fronte ai disastri provocati da guerre, epidemie o calamità naturali. La creazione di questi ospedali servì inoltre per distinguere due gruppi: le donne infermiere e le infermiere professioniste retribuite ${ }^{61}$.

\section{La professionalizzazione delle infermiere}

La messa in funzione delle scuole catalane e di quelle create dalla Croce Rossa costituì un grande passo in avanti per le donne, perchè permise loro di raggiungere un grado di formazione per esercitare un'attività remunerata. Grazie a queste scuole, le infermiere non sarebbero più state le giovani incolte, inesperte, e dalla vocazione filantropica di fine XIX e inizio XX secolo, ma professioniste con un ottimo grado di preparazione, capaci di competere a livello europeo ${ }^{62}$.

In Spagna, l'introduzione di queste scuole significò che le infermiere avrebbero dovuto poter contare con la stessa formazione di cui godevano le parigrado europee. Il processo, anche se lento e contrassegnato da fasi altalenanti, fu inarrestabile. Dal 1863 si ripeterono riunioni periodiche del Comitato Internazionale della Croce Rossa, così come della Conferenza Internazionale della Croce Rossa. Alla Conferenza dell'Aia del 1928, si celebrò una riunione per analizzare quale fosse la formazione delle infermiere. In tutti i paesi in cui era attiva la Croce Rossa, per poter studiare infermieristica le candidate dovevano avere un'età compresa tra i venti e i trentacinque anni ed una buona istruzione generale, oltre a dover sostenere un esame di accesso ${ }^{63}$. Le future infermiere dovevano frequentare un corso preparatorio di tre mesi, più due anni di studi - con lezioni teoriche impartite da medici - e corsi pratici nell'ospedale - tenuti dalle Sorelle della Carità, infermiere diplomate ed abilitate a dirigere tali corsi -, oltre a quelli di aggiornamento. Le studentesse venivano esaminate da un comitato composto dal medico-comandante dell'esercito, dal direttore dell'ospedale e da due medici nominati dalle autorità militari; le persone incaricate di supervisionare la preparazione delle studentesse di infermieristica erano sempre uomini. Come sostiene Carles Clemente nel suo libro, le infermiere formate dalla Croce Rossa si dividevano in tre

${ }^{61}$ Cfr. CLEMENTE, Josep Carles, La Escuela Universitaria de Enfermeras de Madrid, cit., pp. 61-62.

${ }^{62}$ HERNÁNDEZ MARTÍN, Francisca, La enfermería en el siglo XX: De oficio a profesión. Los momentos del cambio, Madrid, Jornada Nacional de Historia de la enfermería, E.U.E. de la Universidad Complutense de Madrid, 1996.

63 HERNÁNDEZ MARTÍN, Francisca, PINAR GARCÍA, María Elena, «La situación hospitalaria en el Madrid del siglo XVIII. El papel de los cuidadores-enfermeros en dichos establecimientos», in La enfermería española. Investigación, Madrid, Escuela Universitaria de Enfermería, Fisioterapia y Podología, Universidad Complutense de Madrid, 1995, pp. 73-90. 
categorie: «le infermiere professioniste che hanno realizzato due anni di studio nell'ospedale della Croce Rossa, le infermiere diplomate che hanno realizzato un anno di servizio supplementare in un ospedale e le infermiere-capo, che hanno realizzato sei mesi di lavoro come direttrici di un pronto soccorso o di un ambulatorio della Croce Rossa» ${ }^{64}$. Secondo l'autore, il gennaio 1927 l'Istituzione delle Donne Infermiere contava 2.998 donne, di cui 944 appartenenti il gruppo de infermiere diplomate che hanno realizzato due anno di servizio supplementare in un ospedale della Croce Rossa e 2.054 al secondo, un anno di servizio supplementare in un ospedale della Croce Rossa, mentre 263 erano religiose e 2.735 non appartenevano ad alcun ordine ${ }^{65}$. Le donne infermiere venivano distribuite tra gli ospedali di quasi tutta la Spagna e furono soggette a diversi cambi di definizione, secondo la legislazione all'epoca vigente. Questi dati possono essere considerati affidabili, vista l'assoluta impossibilità di svolgere questo lavoro saltuariamente in considerazione del fatto che l'impegno richiesto era totale. Il Manual de la enseñanza de enfermeras del 1917 illustra chiaramente queste difficoltà. Le infermiere, professionalizzandosi, consentivano a una platea sempre più ampia di ottenere un lavoro retribuito, che consentisse al mondo femminile di ottenere l'indipendenza economica e l'uguaglianza.

Seguendo le indicazioni del manuale, venne creato il Corpo delle Donne Infermiere al servizio dell'Associazione Croce Rossa, le cui componenti dovevano compiere una missione basata sui concetti di carità ed assistenza ai malati, per cui «l'ausiliare della Croce Rossa ha sì il compito di raccogliere fondi, di aumentare le possibilità dell'Associazione... e sappia amministrare allo stesso tempo, liberamente ma in modo scientifico, il soccorso a feriti e ammalati ${ }^{66_{»}}$. Bontà d'animo e disinteresse personale si dovevano manifestare nel compimento di alcuni obblighi che dimostravano come non si fosse in presenza dell'uguaglianza delle donne, quanto piuttosto vi fosse una forte dipendenza dal medico, al quale dovevano obbedire senza eccezioni, in quanto depositario delle conoscenze delle pratiche sanitarie:

l'infermiera deve limitarsi unicamente ed esclusivamente a compiere il volere del medico, del quale è assistente, senza interpretazioni e giudizi personali che possano risultare lesivi per la salute del malato, e senza perdere di vista quelli che sono i doveri e i diritti di un'infermiera ${ }^{67}$.

${ }^{64}$ Cfr. CLEMENTE, Josep Carles, La Escuela Universitaria de Enfermeras de Madrid, cit., p. 54 .

65 Ibidem, p. 68.

${ }^{66}$ Ibidem, p. 146.

${ }_{77}$ DOMÍNGUEZ-ALCÓN, Carmen, op. cit., p. 152. 
Per ciò che riguardava la dipendenza dal medico, nonostante tutto fosse già piuttosto chiaro, si ricordava che l'infermiera «sarà obbediente e compirà ciò che le si ordina e contribuirà mettendo a disposizione la propria intelligenza»68. Si combinavano il controllo e il riconoscimento delle proprie capacità, ma il suo dovere consisteva comunque nel compiere tali funzioni con amorevolezza, perché «attraverso un carattere amorevole, l’infermiera troverà la fermezza necessaria nei confronti degli ammalati», senza intromettersi nella vita personale dell'ammalato ed in aspetti quali la religione, il modo di pensare, o la politica; su quest'ultimo aspetto, la Croce Rossa era intransigente ed esigeva dalle infermiere la completa neutralità ${ }^{69}$. Un'infermiera della Croce Rossa non poteva dimostrare avversione verso gli ammalati e doveva affrontare ogni situazione, per quanto cruenta fosse, oltre a conoscere i propri doveri in caso di decesso dell'ammalato ${ }^{70}$.

Per la nuova professione si pubblicò anche un Regolamento per il Corpo delle Infermiere Professioniste della Croce Rossa, dove si esplicitavano le caratteristiche che il nuovo corpo doveva riunire. Il documento, elaborato nel 1922 e riportato anche nel libro di Clemente, è un compendio di indicazioni sugli studi di infermieristica, che presenta situazioni talvolta controverse ${ }^{71}$. Il primo capitolo è suddiviso in 18 articoli, ognuno con un contenuto preciso. Il secondo dichiara la costituzione del Corpo di Infermiere Professioniste della Croce Rossa, con il compito di dare istruzioni alla futura infermiera e consentirle di svolgere il proprio lavoro negli ospedali, assistendo gli ammalati direttamente presso il loro domicilio o di mettere in pratica le proprie conoscenze, laddove queste venivano richieste. Il luogo nel quale potevano ricevere questa formazione erano gli ospedali già esistenti, dove avrebbero potuto frequentare i corsi tutte le donne spagnole che lo avessero voluto, tra i venti e i trentacinque anni, e che fossero state in possesso di una serie di requisiti, come dedicarsi a studiare all'interno dell'ospedale costruendo un percorso di studi ed esami e possedere quelle qualità morali esplicitate nel regolamento. In questo terzo articolo appariva una prima ed evidente discriminazione per la donna, dato che le minorenni e le donne sposate dovevano presentare l'autorizzazione necessaria. Il quarto articolo serviva per delineare

${ }^{68}$ CLEMENTE, Josep Carles, La Escuela Universitaria de Enfermeras de Madrid, cit., p. 147. 69 HERNÁNDEZ CONESA, Juana, MORAL DE CALATRAVA, Paloma, ESTEBAN-ALBERT, Manuel, Fundamentos de la enfermería: Teoría y método, Madrid, Mcgraw-Hill, 2002.

$7^{\circ}$ CLEMENTE, Josep Carles, La Escuela Universitaria de Enfermeras de Madrid, cit., p. 148.

${ }^{71}$ REGLAMENTO PARA EL CUERPO DE ENFERMERAS PROFESIONALES DE LA CRUZ ROJA E INSTRUCCIONES PARA LA ADMISIÓN DE ALUMNAS ASPIRANTES A INGRESO EN EL MISMO: CURSOS Y ENSEÑANZAS in CLEMENTE, Josep Carles, La Escuela Universitaria de Enfermeras de Madrid, cit., pp. 151-169. Il documento del 1922, proviene dal Servicio de Documentación de la Oficina General de la Cruz Roja Española. 
le differenze tra la donna infermiera e l'infermiera professionale. Secondo quanto vi veniva affermato, "L'Infermiera Professionale appartiene ad un Corpo dipendente dall'Assemblea Centrale delle Signore Della Croce Rossa, le cui disposizioni deve riconoscere e mettere in pratica»; se ne evince, pertanto, che le infermiere professioniste e le donne infermiere costituivano due corpi distinti, e che il primo dipendeva dal secondo. Secondo il regolamento la distinzione era dovuta ad una differenza di spirito corporativo (se una donna infermiera desiderava entrare come allieva, perdeva il titolo). Inoltre, nel caso in cui si fossero trovate a lavorare insieme per una qualche evenienza, l'infermiera professionale doveva sempre riconoscere l'autorità della donna infermiera. L'articolo successivo imponeva alle infermiere professioniste di prestare servizio presso l'ospedale che avesse avuto bisogno della loro opera, sia che questo fosse della Croce Rossa, della Sanità Militare o un qualsiasi ospedale civile o locale ${ }^{72}$. Sempre secondo il regolamento, le infermiere professioniste erano obbligate ad ubbidire alle indicazioni del personale medico e a mostrare disciplina, subordinazione e rispetto verso i responsabili della clinica e i medici direttori degli ospedali. Gli altri articoli si dedicavano alle questioni formali relative al documento di lavoro, che tutte le infermiere professioniste dovevano possedere per potervi annotare i servizi prestati, le procedure di realizzazione, la buona condotta, etc. Nell'eventualità in cui avessero voluto lavorare privatamente o per strutture che ne avessero avuto bisogno, la richiesta doveva passare attraverso la Giunta delle Signore degli Ospedali, o anche arrivare direttamente alle infermiere, che in tal caso potevano riscuotere il salario corrispondente. Questo gruppo di infermiere professioniste poteva essere supervisionato dall'Ispettore generale; queste erano tenute a comunicare sempre i cambi di residenza o di luogo di lavoro alla segreteria della Giunta dell'Ospedale corrispondente o della Giunta Locale dell'Associazione ${ }^{73}$. Tanti formalismi rendevano il gruppo un collettivo regolato in modo autoritario, in cui erano chiaramente rispettate le gerarchie delle istituzioni che lo dirigevano.

In un altro articolo venivano specificati quali fossero l'uniforme ed il distintivo richiesti: «L'uniforme sarà la stessa approvata per le Donne Infermiere; le alunne porteranno una piccola croce rossa sulla parte frontale della cuffia, che si manterrà anche una volta raggiunto il Titolo ufficiale, e verrà integrato opportunamente con un altro distintivo». Un articolo spiegava anche quali erano i premi e le punizioni: i premi

${ }^{72}$ HERRERO LÓPEZ, Maria Milagros, CUELLO RUBIO, Jacinto, Evolución histórica de la formación básica de enfermería desde el año 1951-87, in I Jornadas de Enfermería, Avances de la enfermería en el siglo XX, Madrid, Ministerio de Sanidad y Consumo, 1998, pp. 19-33.

73 MARTÍNEZ NAVARRO, J. Ferran, «Factores sociales en el origen de la enfermería en España», in Revista de Sanidad e Higiene Pública, 4, 1975, pp. 343-356. 
consistevano in menzioni onorifiche, medaglie ${ }^{74}$ o qualsiasi altro segno distintivo a discrezione dell'Assemblea Generale, mentre le punizioni erano ammonizioni, deplorazioni o sospensioni per sei mesi, mentre in caso di recidività si poteva aprire un'indagine o addirittura poteva scattare l'allontanamento dal Corpo, se così veniva deliberato dall'Assemblea Generale ${ }^{75}$.-L'iscrizione implicava non solo un impegno da parte dell'iscritta, ma anche dei genitori, che rispondevano del suo impegno nell'adempimento dei servizi come infermiera. Anche in questo caso, la burocrazia non poteva mancare: «consegneranno anche copia del certificato di nascita corrispondente all'iscrizione al Registro Civile, copia del documento di battesimo e certificato di buona condotta; autocertificheranno di avere le condizioni educative e culturali necessarie, e si sottoporranno ad un esame preventivo per verificarne l'istruzione base, mentre un altro medico verificherà l'idoneità fisica» ${ }^{76}$. Come si può notare era un quadro completamente diverso rispetto a quello dell'epoca delle prime infermiere, analfabete e povere.-Negli ospedali-scuole queste erano in possesso di un documento di iscrizione sul quale venivano segnalati tutti i dati relativi al loro lavoro nelle aule, con la controcertificazione della Presidentessa della Giunta dell’Ospedale.

\section{Infermiere e professoresse}

Nonostante la nascita delle scuole e la regolarizzazione dell'insegnamento, le infermiere spagnole avevano davanti ancora molti ostacoli da superare prima di vedere riconosciute le proprie capacità. L'esempio che più chiaramente riflette la posizione di inferiorità che occupavano nell'ambito sanitario, è dato dalle difficoltà che incontravano per diventare docenti nelle scuole di infermieristica. Tra i progressi ottenuti dalle infermiere durante il processo formativo, c'è un dato significativo che evidenzia il controllo a cui le studentesse erano sottoposte. In una prima fase, fino alla fine del secondo decennio del XX secolo, l'insegnamento era impartito da medici che ricevevano una specifica formazione, mentre le infermiere venivano istruite per aiutare i medici stessi ed eseguirne gli ordini. Negli anni Quaranta, i medici continuavano ad

\footnotetext{
74 É opportuno ricordare che la medaglia più importante che un'infermiera della Croce Rossa potesse ottenere, era la «Medaglia Florence Nightingale», istituita in ricordo della vita e dell'impegno di Florence Nightingale, e assegnata alle infermiere che si distinguevano nell'assistenza a feriti ed ammalati. Il regolamento della sua istituzione si può trovare in CLEMENTE, Josep Carles, La Escuela Universitaria de Enfermeras de Madrid, cit., pp. 283286.

75 CLEMENTE, Josep Carles, La Escuela Universitaria de Enfermeras de Madrid, cit., pp. 152154. Il regolamento fu approvato da un Regio Decreto del Ministero di Guerra, il 26 ottobre 1922 (R.O. núm. 242).

${ }^{76}$ DOMÍNGUEZ-ALCÓN, Carmen, op. cit., p. 152.
} 
essere i responsabili dell'insegnamento delle infermiere, ma si cominciava a lasciar spazio a una seppur minima partecipazione femminile per le infermiere, che, oltre all'assistenza ai medici, avevano un ruolo di intermediazione tra questi ed i pazienti. Nel primo quarto di secolo compaiono nuove e prestigiose scuole, come la Cruz Roja Española, sia a Madrid che a Barcellona, la Casa de la Salud Marqués de Valdecilla a Santander, e Santa Madrona a Barcellona. Le condizioni di ammissione alle stesse seguivano i criteri stabiliti da Nightingale y Kaiserswerth, precursori dell'Infermieristica moderna in Europa. Le levatrici avevano invece le proprie scuole, vincolate alle strutture dedicate alla maternità. Per quanto riguarda infine i tirocinanti, questi si preparavano presso le Facoltà di Medicina.

Ogni percorso seguiva un cammino diverso, così come distinto era il titolo conferito. Tuttavia, tra i diversi piani e programmi esistevano evidenti coincidenze riguardo il corpus delle materie affrontate. Fino al 1940, gli studi precedenti richiesti erano quelli primari, sia per tirocinanti che per infermiere. Il temario teorico-pratico era praticamente lo stesso: settanta temi per le infermiere, e settantotto per i tirocinanti. La diversa focalizzazione degli studi di praticante e infermiera, influiva sulla successiva scelta dell'area nella quale avrebbero esercitato l'uno e l'altra. I primi si muovevano soprattutto in ambito extraospedaliero, mentre le seconde all'interno degli ospedali. Le prime Reales Órdenes, la cui funzione consisteva nel regolare l'esercizio della professione, risalgono agli ultimi anni del secolo XIX77. Già nell'anno 1941 si stabiliscono per Orden Ministerial le competenze delle infermiere, evidenziando chiaramente che l'ambito nel quale potevano sviluppare le proprie funzioni era in seno a istituzioni di tipo religioso e patriottico, ed esplicitando il programma ufficiale per Infermiere ${ }^{78}$.

Per ciò che riguarda le responsabilità, vengono indicate l'assistenza agli infermi, la pulizia, l'alimentazione, la raccolta di dati clinici e la somministrazione di medicine. Più tardi, nel 1945, per Orden Ministerial si determinò che «Le infermiere in possesso di un titolo ufficiale erano autorizzate e considerate idonee:

1. All'assistenza di tipo famigliare, la pulizia, l'alimentazione, la raccolta di dati clinici e la somministrazione di medicine agli infermi. Questi servizi potranno essere realizzati tanto in seno alle istituzioni di tipo assistenziale come presso il

\footnotetext{
77 Real Orden, in approvazione del Reglamento para las carreras de Practicante y Matronas. Ministerio de Fomento (Madrid), 16 novembre 1888, pubblicato il giorno 18.

78 Programma del 6 luglio 1941 (M. Educación Nacional), BOE, 16 luglio 1941. Il Ministerio de Educación Nacional, promulgò l'Orden del 4 agosto 1953 riguardo le Escuelas de Enfermeras e le Normas de Estudio.
} 
domicilio dell'ammalato.

2. All'assistenza di operazioni chirurgiche, interventi e cure, a supporto dei medici»79.

Tali dichiarazioni ufficiali rappresentano un chiaro esempio dell'istituzionalizzazione e tecnicizzazione dell'Infermieristica, riflettendo la realtà professionale del tempo e i progressi scientifici. Nell'anno 1944 si creano i Colegios Oficiales de Auxiliares Sanitarios e il Consejo General de Colegios, il cui compito era quello di ospitare tirocinanti, infermiere e levatrici. Il Consejo General, costituito dall'insieme dei Colegios Provinciales, massimo organismo professionale e di rappresentanza, era strutturato in tre sezioni, corrispondenti ai tre titoli esistenti. Presso i Colegios Provinciales si osservava la stessa organizzazione, che di conseguenza rifletteva nella pratica l'esistenza dei tre profili professionali distinti. Nel 1945, la legislazione obbligò tanto infermiere, come tirocinanti e levatrici, a riunirsi in collegi per esercitare la propria professione.

Negli anni Cinquanta si produssero molti cambiamenti nei programmi di insegnamento, che tuttavia si tradussero in pochi effetti concreti; le infermiere cominciano comunque a partecipare nel ruolo di istruttrici all'insegnamento clinico. Fu negli anni Settanta che le infermiere ebbero finalmente accesso all'insegnamento teorico, mantenendo il quasi completo controllo degli insegnamenti pratici nelle cliniche. Negli anni Ottanta, anche se le funzioni non erano ancora completamente delineate, si può affermare che raggiunsero uno degli obiettivi principali tra quelli che inseguivano, dato che sempre più infermiere furono ammesse esercitare come professoresse titolate nelle Scuole Universitarie di infermieristica. Questo studio ha voluto presentare l'evoluzione della figura dell'infermiera spagnola durante i secoli XIX e XX ed il cammino intrapreso verso il suo riconoscimento come professionista. In questo sforzo dovette affrontare i pregiudizi della classe politica spagnola, incapace di fornirle una formazione culturale e di evitare discriminazioni sessiste, ma allo stesso tempo poté contare sull'aiuto e l'appoggio di alcune istituzioni importanti, come la monarchia o la Croce Rossa, fondamentali per il raggiungimento di un titolo accademico, il definitivo distanziamento dall'ambito del filantropismo e la sua trasformazione di questa figura in quella di una professionista dell'ambito sanitario.

79 Orden del 26 noviembre 1945, con il quale si dichiarano idonee e si autorizzano le infermiere in possesso del titolo ufficiale. 


\section{* L'autore}

Maria Ángeles Hijano Pérez ha conseguito il Dottorato di ricerca presso l'Universidad Autónoma de Madrid, con una tesi intitolata El Gobierno municipal de la Corona de Castilla: del Antiguo Régimen a la revolución liberal sotto la direzione di Miguel Artola Gallego. È titolare dell'insegnamento di Storia contemporanea nella stessa Università e ha participato a numerosi progetti di ricerca. Tra le sue pubblicazioni: Los ciclos revolucionarios: 1820-1830-1848 (Madrid, EUDEMA, 1992); El Pequeño Poder: El Municipio en la Corona de Castilla, Siglos XV Al XIX (Madrid, Fundamentos, 1992); Victoria Eugenia de Battenberg: una reina exiliada, 1887-1969 (Madrid, Alderabán, 2000); Las ordenanzas municipales como norma del gobierno local (Granada, Centro de Estudios Municipales y de Cooperación Internacional, 2001); Las revoluciones europeas de 1820 a 1848 (Madrid, Dilema, 2012).

URL: < http://www.studistorici.com/progett/autori/\#HijanoPerez >

\section{Per citare questo articolo:}

HIJANO PÉREZ, Maria Ángeles, «ll cammino delle infermiere verso la professionalizzazione», Diacronie. Studi di Storia Contemporanea : Spazi, percorsi e memorie, 29/10/2013,

URL:< http://www.studistorici.com/2013/10/29/hijano-perez_numero_15/ >

Diacronie Studi di Storia Contemporanea $\beta$ www.diacronie.it

Risorsa digitale indipendente a carattere storiografico. Uscita trimestrale. redazione.diacronie@hotmail.it

Comitato di redazione: Marco Abram - Jacopo Bassi - Luca Bufarale - Alessandro Cattunar - Elisa Grandi - Deborah Paci - Fausto Pietrancosta - Matteo Tomasoni - Luca Zuccolo

Diritti: gli articoli di Diacronie. Studi di Storia Contemporanea sono pubblicati sotto licenza Creative Commons 2.5 Possono essere riprodotti a patto di non modificarne i contenuti e di non usarli per fini commerciali. La citazione di estratti è comunque sempre autorizzata, nei limiti previsti dalla legge. 